\title{
Disclosing errors that affect multiple patients
}

\author{
Roger Chafe PhD, Wendy Levinson MD, Terrence Sullivan PhD
}

$\mathrm{O}$ rganizations in a number of countries have developed guidelines to help health care providers and institutions disclose medical errors to patients. ${ }^{1-4}$ The primary focus of such guidelines is the disclosure of errors that affect individual patients. Yet many adverse events involve hundreds, if not thousands, of patients. This is particularly the case when errors are related to equipment failure, the performance of medical procedures or the interpretation of diagnostic procedures..$^{5-8}$ The basic principle that patients should be informed quickly, fully and respectfully of errors that adversely affect them holds true regardless of the number of patients involved. ${ }^{9}$ But timely and complete disclosure is especially complex when dealing with errors affecting large numbers of patients and raises challenges well beyond those of identifying who is affected.

When an error involves only 1 patient, the fact that a problem has occurred and the identity of the affected patient are often immediately apparent. But errors that affect large numbers of patients involve more stakeholders, usually occur over longer periods, risk greater breaches of public trust in the affected institutions and require more comprehensive communication strategies. Institutional errors involving many patients, therefore, cannot be handled in the same way as those involving individual patients.

\section{Steps to addressing an error}

Based on our experience, we have identified 6 steps that health care organizations generally need to work through when addressing errors that involve many patients (Box 1). The timely identification of an error is one of the more difficult steps. Errors are repeated because they are not immediately apparent. Typically, concerns are first raised by a clinician, technician or patient. Based on an initial estimate of the number of people affected, an appropriate sample of records or procedures needs to be reviewed to determine the extent of any further investigation.

If a full review is necessary, then the next challenge facing the institutional leadership is to establish the period of the adverse event and the scope of the review. This step involves identifying a project team and determining the resources needed to undertake a full review. A list of patients who may have been affected by the error needs to be developed and their records included in the review. Staff need to be assigned and trained to perform high-quality and painstaking audits. Reliability checks and quality controls need to be considered in the training phase if more than 1 staff member is assigned to this task.

When a laboratory or diagnostic testing error has occurred,

\section{Key points}

- Guidelines for disclosure of medical errors in Canada and other countries do not provide adequate recommendations for addressing errors that affect multiple patients.

- Health care organizations face institutional barriers to the timely and complete disclosure of such errors.

- Six steps are proposed for health care organizations to follow when addressing adverse events involving large numbers of patients.

- Government agencies, professional associations and accreditation bodies could help health care organizations in adopting policies and developing protocols for such disclosure.

the review process needs to introduce steps to determine whether the resulting diagnostic report affected the decisionmaking of the clinician, the appropriateness of the patient care that was delivered and the potential outcomes of that care. Any decisions related to patient care that were made by the clinician on the basis of such a report need to be reviewed by an expert clinician or health care team. Moreover, institutional leaders need to determine the levels of independence required for those who make such evaluations as well as those who evaluate the overall findings of a review. Finally, physicians need to follow up with affected patients who require changes in treatment or have questions about their care.

Another difficult step is determining how and when to inform patients, other affected hospital personnel, external authorities, insurers and other stakeholders such as family physicians, other health care institutions and the public. Often numerous hospital departments need to be involved. Given the workload of the task, conducting a timely review may even require assistance from other health care organizations. Depending on the number of patients involved, months may be needed to determine whether specific patients were affected. Locating and contacting a large number of former patients is not easy. We believe that a disclosure process on this scale should not be initiated or led by the physicians who were directly involved in the error, despite current recommendations to the contrary. ${ }^{2}$

A clear disclosure plan is a necessity. The plan needs to include a timeline with specific dates and a communication strategy for disclosure to all affected groups, including the

From Cancer Care Ontario (Chafe, Sullivan), Sunnybrook Health Science Centre (Levinson), the Department of Health Policy, Management and Evaluation (Chafe, Sullivan) and the Department of Medicine (Levinson), University of Toronto, Toronto, Ont. 
Box 1: Proposed steps to addressing a medical error affecting multiple patients

1. Identify the error in a timely fashion. Typically this step is taken because of concern raised by patients or medical personnel about the quality of care given to a group of patients.

2. Conduct a review of an appropriate sample of records or procedures to determine the extent of the error.

3. If a full review is necessary, identify a project team, establish the scope of the review and determine the resources needed for the review.

4. Identify patients who may have been affected by the error and include their records in the review.

5. Review clinical records. When an error in laboratory or diagnostic testing has occurred, determine whether the resulting diagnostic report affected the clinician's decisions, the appropriateness of care provided and the outcomes of that care.

6. Inform patients and other stakeholders (e.g., affected hospital personnel, external authorities, insurers, family physicians, other health care institutions and the public). Affected patients need to be followed up individually by the physician who has been most closely involved in managing their care.

public. The specific information to be provided and the names of those who will provide it need to be listed.

A strategy for the management of data is also imperative. The team responsible for carrying out the review process needs to be able to identify, in real time, when all successful and unsuccessful attempts have been made to contact each of the patients affected and other stakeholders. To ensure appropriate sensitivity to the families of affected patients who have since died, protocol needs to be developed outlining the timing and manner of disclosure in these cases. Patients who are incapacitated may need to be contacted through surrogate decision-makers, who will need to be identified.

\section{Barriers to disclosure}

Medical institutions are not generally disposed to disclosing errors. When large numbers of patients are involved, the disclosure process is time- and resource-intensive. Several systemic factors can also jeopardize the process, minimize it or simply slow it down.

Health care administrators may feel uncomfortable disclosing a medical error before all of the facts are known. They may even receive legal advice to delay disclosure so as not to expose the organization to greater liability. The situation may be complicated by real or perceived political pressures to limit communication about the error generally or to shift blame to specific people. An adverse event may involve several departments within a hospital, or it may involve the staff of both a health care organization and the government department responsible for the organization. In these cases, no one may take full responsibility for the error, and the need for timely disclosure to affected patients can fall through the cracks.
Still, moving quickly to publicly disclose an error has clear benefits, even when cause has not yet been determined. When the Ottawa General Hospital disclosed in 2008 that 326 patients with skin cancer had been given lower doses of radiation than they should have received, an editorial in the Ottawa Citizen concluded that the very act of disclosure, along with the public apology, "helped ameliorate some of the problems the hospital created." 10 The patients who were affected by that error launched a class-action lawsuit against the hospital. ${ }^{11} \mathrm{On}$ balance, early disclosure may lower the risk of litigation, as shown by documented cases of disclosure of errors involving individual patients in the United States. ${ }^{12,13}$

Other industries have faced the challenges involved in contacting large numbers of people after a major error. Companies that make food products have encountered such situations when bacterial contamination has occurred. ${ }^{14}$ Although health care organizations face unique challenges when major errors occur, including the need to contact patients individually and the need to comply with rules that govern the profession, decision-makers in health care organizations can learn from others. A case in point is the rapid action in 2008 by Maple Leaf Foods Chief Executive Officer Michael McCain to apologize and assume full responsibility for the health consequences of contamination by Listeria at one of its processing plants. ${ }^{15}$ The company also provided continual updates on its review to the public.

\section{Topics for guidelines}

We believe that national guidelines for addressing large-scale errors would help organizations overcome the institutional barriers to timely disclosure. Such guidelines need to address several questions. When should a health care organization inform its patients about such an error and when should it inform the public? What information should be provided to patients, particularly when the patients affected have not yet been identified and the cause and impact of the problem are not yet known? How should the process of disclosure be managed procedurally and ethically?

As a starting point, we propose that several practices be adopted by health care organizations (Box 2). After an error affecting numerous patients has been confirmed, it needs to be disclosed in a timely manner, even if its extent and full impact are not yet clear. This precaution is in keeping with recommended practices in crisis communication ${ }^{16}$ and with what the public likely expects from public institutions. The organization itself needs to be the first to present the information to the public. Transparency needs to trump concerns about increasing legal liability.

Initial disclosure needs to avoid identifying individual clinicians or making assumptions about cause. All patients who may have been harmed by the error need to be followed up individually. Those who may require clinically important adjustments to therapy or may have been seriously injured need to be given priority and followed closely. Organizations would be well advised to prepare a clear communication strategy that outlines the organization's plan for communicating with the media and public during the crisis. The establishment of a dedicated information phone line or website needs 
Box 2: Proposed guidelines for disclosing a medical error affecting multiple patients

- After an error has been confirmed, it should be disclosed in a timely manner, even if its extent or full impact is not yet clear.

- Initial disclosure should not identify individual clinicians or include assumptions about cause.

- All patients who may have been harmed by the error should be followed up individually.

- A communications plan should be established that includes mechanisms (e.g., a dedicated phone line or website) for affected patients to gain access to information and ask questions.

- Affected patients should be given priority over current patients when appropriate.

- An analysis of the root cause should be conducted and led externally.

- The results of the analysis should be released publicly with statements of the actions undertaken to address problems identified.

- A clear and fair process should be used to evaluate the performance of clinicians involved in the adverse event.

to be considered for use by affected patients who have ongoing concerns and information needs.

The disclosure of a large-scale medical error is not a single event, but rather an ongoing process. Patients and the public need to be updated as the process of disclosing and addressing related problems continues. A full — and optimally external — analysis needs to be conducted to identify cause. A review needs to focus on the actions necessary to ensure quality of care, rather than on punitively identifying individual health care providers. A report on the underlying causes of the error and the changes undertaken by the organization to address those causes needs to be released to the public. Finally, a clear and fair protocol needs to be established for reviewing the performance of clinicians involved in the medical error, especially in the area of interpretive diagnostics, where standards of accuracy depend on peer review.

\section{Conclusion}

National guidelines alone may not ensure that health care organizations overcome institutional barriers to disclosure of medical errors. We propose, therefore, that governments and health care associations assist individual organizations with establishing policies for early disclosure of large-scale ad- verse events and with developing protocols for identifying affected patients. Institutional accreditation bodies are well positioned to play a key role by requiring that organizations adopt such policies.

\section{Competing interests: None declared.}

Contributors: All of the authors contributed to the conception, design and drafting of the article and approved the final version submitted for publication.

Acknowledgements: We thank Dr. Robert Bell and Dr. Mark Dobrow for their comments on earlier drafts of this article.

\section{REFERENCES}

1. Australian Commission on Safety and Quality in Health Care. Open disclosure standard: a national standard for open communication in public and private hospitals following an adverse event in healthcare. Sydney (Australia): The Commission; 2008. Available: www.safetyandquality.gov.au/internet/safety/publishing.nsf /Content/3B994EFC1C9C0B22CA25741F0019FDEE/\$File/NOD-Std \%20reprinted\%202008.pdf (accessed 2009 Jan. 7).

2. Canadian Patient Safety Institute. Canadian disclosure guidelines. Ottawa (ON): The Institute; 2008. Available: www.patientsafetyinstitute.ca/uploadedFiles /Resources/cpsi_english._april28.pdf (accessed 2009 Jan. 7).

3. National Patient Safety Agency. Safer practice notice: being open when patients are harmed. London (UK): The Agency; 2005. Available: www.npsa.nhs.uk/nrls /alerts-and-directives/notices/disclosure/ (accessed 2009 Jan. 7).

4. National Quality Forum. Safe practices for better healthcare. Washington (DC) The Forum; 2008. Available: www.qualityforum.org/projects/completed/safe _practices/ (accessed 2009 Jan. 7).

5. Adhopia V. Misdiagnosed: anatomy of Newfoundland's cancer-testing scandal CBC News [Toronto]. 2008 Apr. 28. Available: www.cbc.ca/news/background /cancer/misdiagnosed.html (accessed 2009 Jan. 7).

6. Morris C. N.B. pathologist tells inquiry he wants to get back to work. Globe and Mail [Toronto] 2008 May 30. Available: www.theglobeandmail.com/servlet/story /RTGAM.20080530.wpathologist0530/BNStory/National/home (accessed 2009 Mar. 4).

7. Another hospital contacts patients after diagnostic errors discovered. National Post [Toronto] 2008 May 1;Sect A:8.

8. Furlow B. Radiotherapy errors spark investigation and reform. Lancet Oncol 2009;10:11-2.

9. Gallagher TH, Studdert D, Levinson W. Disclosing harmful medical errors to patients. N Engl J Med 2007;356:2713-9.

10. Sorry actually matters [editorial]. Ottawa Citizen 2008 Apr. 24;Sect C:4

11. Canadian Press. Class-action suit seeks $\$ 30 \mathrm{M}$ for radiation errors. Globe and Mail [Toronto]. 2008 Dec.19;Sect A:9.

12. Kraman SS, Hamm G. Risk management: extreme honesty may be the best policy. Ann Intern Med 1999;131:963-7.

13. Clinton HR, Obama B. Making patient safety the centerpiece of medical liability reform. N Engl J Med 2006;354:2205-8.

14. Product recall procedures. Ottawa $(\mathrm{ON})$ : Health Canada. Health Products and Food Branch; 2008. Available: www.hc-sc.gc.ca/dhp-mps/alt_formats/hpfb-dgpsa /pdf/compli-conform/recall_proc-marche_retrait-eng.pdf (accessed 2009 Jan. 7).

15. Maple Leaf Foods. News releases. Toronto (ON): Maple Leaf Foods; 2008. Available: http://investor.mapleleaf.ca/phoenix.zhtml?c=88490\&p=irol-news2008 (accessed 2009 Jan. 7).

16. Seeger M. Best practices in crisis communication: an expert panel process. $J$ Appl Commun Res 2006;34:232-44.

Correspondence to: Dr. Roger Chafe, Cancer Care Ontario, Cancer Services and Policy Research Unit, 620 University Ave., Toronto ON M5G 2L7; fax 416 971-6888;

roger.chafe@cancercare.on.ca 